\title{
Massive intraperitoneal hemorrhage in patients with COVID-19: a case series
}

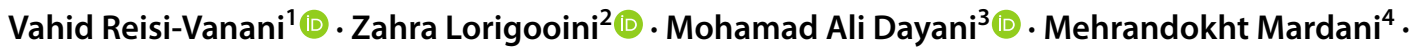 \\ Fereidoun Rahmani ${ }^{4}[$
}

Accepted: 13 April 2021 / Published online: 28 April 2021

(c) The Author(s), under exclusive licence to Springer Science+Business Media, LLC, part of Springer Nature 2021

\begin{abstract}
Coronavirus disease (COVID-19) initiates several life-threatening complications including coagulopathies with a unique characteristic that made this problem challenging. Here we presented 4 cases of RT-PCR positive patients that have experienced deadly intraperitoneal hemorrhage with fourth WHO Bleeding Grade after overcoming their respiratory phase. COVID-19 could induce several coagulopathies with different features that besides iatrogenic interventions increases its mortality and morbidity due to lack of clinical evidence based on well-designed randomized clinical trials on anticoagulation therapies (AT) and administration of varieties of newly approved and non-approved medicines. This report showed the urgent need for investigation on the pathophysiology of COVID-19-associated coagulopathy esp. in hemorrhagic events which are needed to make the best therapeutic decision.
\end{abstract}

Keywords COVID-19 · Coagulopathy · Disseminated intravascular coagulation · Thrombotic microangiopathy

\section{Highlights}

- COVID-19-induced coagulopathies have a unique characteristic, need their specific care.

- Internal bleeding has non-specific signs and symptoms making the early diagnosis difficult.

- Urgent need for research on the pathophysiology of these coagulopathies and clinical research on best choices, dosage, and duration of anticoagulant therapy is needed.

Fereidoun Rahmani

frahmani@skums.ac.ir

1 Student Research Committee, Shahrekord University of Medical Sciences, Shahrekord, Iran

2 Medical Plants Research Center, Basic Health Sciences Institute, Shahrekord University of Medical Sciences, Shahrekord, Iran

3 Department of Radiology, Faculty of Medicine, Shahrekord University of Medical Sciences, Shahrekord, Iran

4 Department of Infectious Disease, Clinical Research Development Unit, Hajar Hospital, Shahrekord University of Medical Sciences, Shahrekord, Iran

\section{Background}

Coronavirus disease (COVID-19) mortality and its association with coagulopathies raised many questions about its pathogenesis. Similarities and differences of these coagulation disturbances with other problems from disseminated intravascular coagulation to antiphospholipid syndrome (APS) and atypical hemolytic uremic syndrome (aHUS) show that COVID-19 coagulopathies have unique characteristics [1]. Although anticoagulation therapies (AT) showed a reduction in mortality of disease this also increased major bleeding events in patients raising the question about the dosage and way of anticoagulation therapies [2]. There are few investigations about bleeding and its risk factors in COVID-19 but hyper fibrinolytic state, thrombocytopenia, coagulation consumption, hypoxia, cytokine storm, direct invasion of the virus, and AT are supposed to increase the incidence of bleedings. Although there are some reports about the occurrence of bleeding in COVID-19 from ICH and GI bleeding to focal kidney hemorrhage [3], this is the first case series of massive deadly intra-abdominal hemorrhage in COVID-19 patients. 


\section{Case presentation}

\section{Case 1}

A 67-year-old woman with a history of several underlying diseases (Table 1) was admitted to the emergency department with anorexia, Nausea, diarrhea, weakness, lethargy, nonproductive cough, and myalgia. In the physical exam, she had stable vital signs, fine crackle in the base of the lungs, and oxygen saturation of $81 \%$ on ambient air that was raised to $92 \%$ with a non-rebreather mask. Radiologic findings revealed bilateral infiltration of lungs with ground-glass opacity; she also had low GFR and a suspected case of acute renal failure (creatinine: $1.5 \mathrm{mg}$ / dl, GFR: $36 \mathrm{~mL} / \mathrm{min} / 1.73 \mathrm{~m}^{2}$ ).

Antiviral therapy with atazanavir-lopinavir, antibiotic therapy with azithromycin and ceftriaxone, famotidine, heparin, dexamethasone, and other conservative therapies had been started for her. The following days, dizziness besides decreases in oxygen saturation and diffuse course crackles of the lungs made us change her dexamethasone to methylprednisolone pulse, and add furosemide, Interferon $\beta 1 \mathrm{a}$, to her therapeutic regime, and start oxygen therapy with BiPAP and non-rebreather mask with an alternating schedule. She also had normal heart function and no signs of pulmonary embolism (PTE). During her hospitalization, although different methods were used for controlling her blood sugar (Bs), we were not successful in this job even by administering a high dose of insulin.

Six-day after hospitalization, he had an electrolyte imbalance that had been corrected and as we were short of atazanavir-lopinavir, we replaced it via lopinavir-ritonavir. The patient's clinical course was slowly improving to the 14th day of hospitalization but because of 'slow' improvements and possible nosocomial infection, the on-call physicians changed her therapeutic regime; furosemide and ceftriaxone had been stopped and piperacillin-tazobactam, linezolid, montelukast, and seroflo spray had been started. Two days later heparin and lopinavir-ritonavir were replaced via enoxaparin and favipiravir; the next day, the patient's clinical course changed a lot, her urine output was decreased and in the physical exam, she had a bulging abdomen with a decreased level of conciseness (Glasgow Coma Scale: 10) and $8 \mathrm{~h}$ later her cell blood count (CBC) showed a severe decrease in hemoglobin. She did not have hematuria, melena, rectorrhagia, hemoptysis, hematochezia, and even in stool exam, there was no occult blood. Her heart function was still normal due to portable echocardiography and in her peripheral blood smear (PBS) few hyper-segmented polymorphonuclear leukocyte (PMN) was seen with anisopoikilocytosis, hypochromic, polychromic, and microcytic RBCs with a decrease in platelet count and no schistocytes. She received 2 pack cell units, and in portable abdominal sonography, she had the echogenic area in her gall bladder and free fluid around the liver; these observations were approved with abdominal CT-Scan with contrast representing mild pleural effusion in both of her lungs, hemoperitoneum around her liver and spleen and retro hemoperitoneum in posterior renal space, left lateroconal fascia, in both of her psoas muscles with forwarding displacement of the left kidney (right < left) extending to iliacus muscle (Fig. 1a).

After stabilizing the patient she was delivered to ICU. In there she received 2 more units of pack cells and the free fluid around the liver was tapped with a sonography guide which confirmed her internal bleeding. The next days she received 2 more pack cells and while it seems that decreases in hemoglobin were stopped she had been died due to cardio-pulmonary arrest.

\section{Case 2}

A 66-year-old woman was admitted due to her malaise and weakness. In physical exam and history taking she was a febrile woman $(\mathrm{T}=38.2)$, stable in vital signs, and clear respiratory sounds complaining of constipation. After hospitalization her viral pneumonia caused us to increase her corticosteroids on her 7 th day of hospitalization and take care of her in ICU. Three days later she was discharged from ICU and her general condition was getting better, but 2 days later and after she had increased her physical activity by walking about $4 \mathrm{~m}$, chest pain was referred to her right upper limb with a reduction in blood pressure caused us to limit his activities to complete bed rest, stop her thiazide drugs and rule out myocardial infarction and myocarditis; she also had problems in urination and abdominal pain mainly in her right lower quadrant that made us take a KUB X-ray from her representing no mechanical blockage in the GI system, presence of fecaloma in right and left colon and the bones with decreased density. On the morning of the next day, Cullen's sign appeared in her umbilical region. Her pallor besides reduction in her blood pressure made her a critically ill patient; she also complained of a feeling of urinary urgency and pressure in her bladder while she was oliguria. In echocardiography, there was not any sign of cardiogenic shock and after a while, we noticed that she had a reduction in her hemoglobin from 11.2 to 9.6. In portable sonography, she had an $800 \mathrm{~cm}^{3}$ hypo echo area in her pelvic and $50 \mathrm{~cm}^{3}$ in her abdominal wall. After stabilizing her with transfusion of pack cell and hydration we have taken her pelvic and abdominal CT-Scan with IV contrast (representing free fluid containing debris in favor of hemoperitoneum surrounding the liver and spleen. Large hematoma in the pelvic cavity (163* $141 \mathrm{~mm})$. Right, rectus muscle sheath hematoma (41 * $68 \mathrm{~mm}$ ) and right oblique and transversalis muscle sheath 


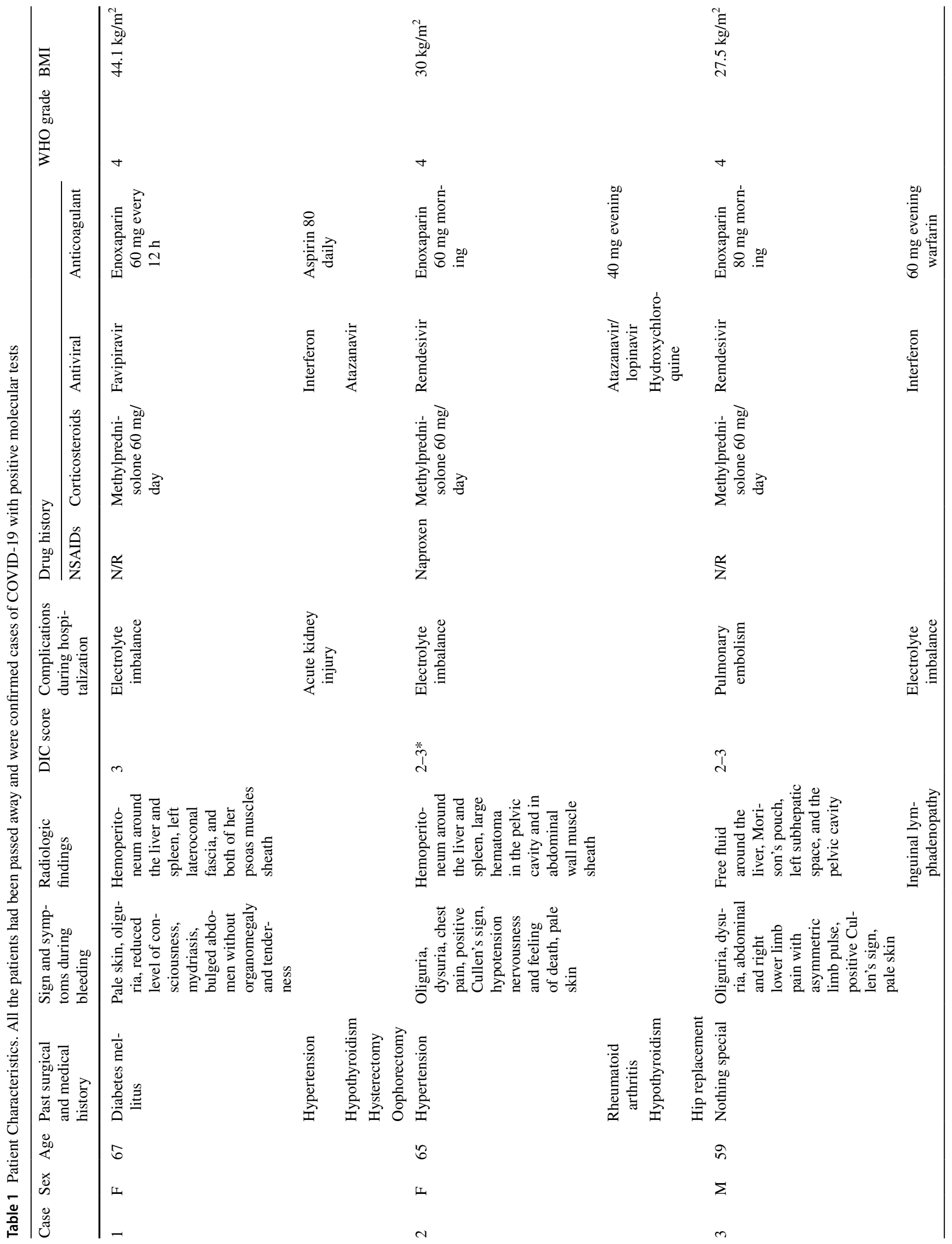




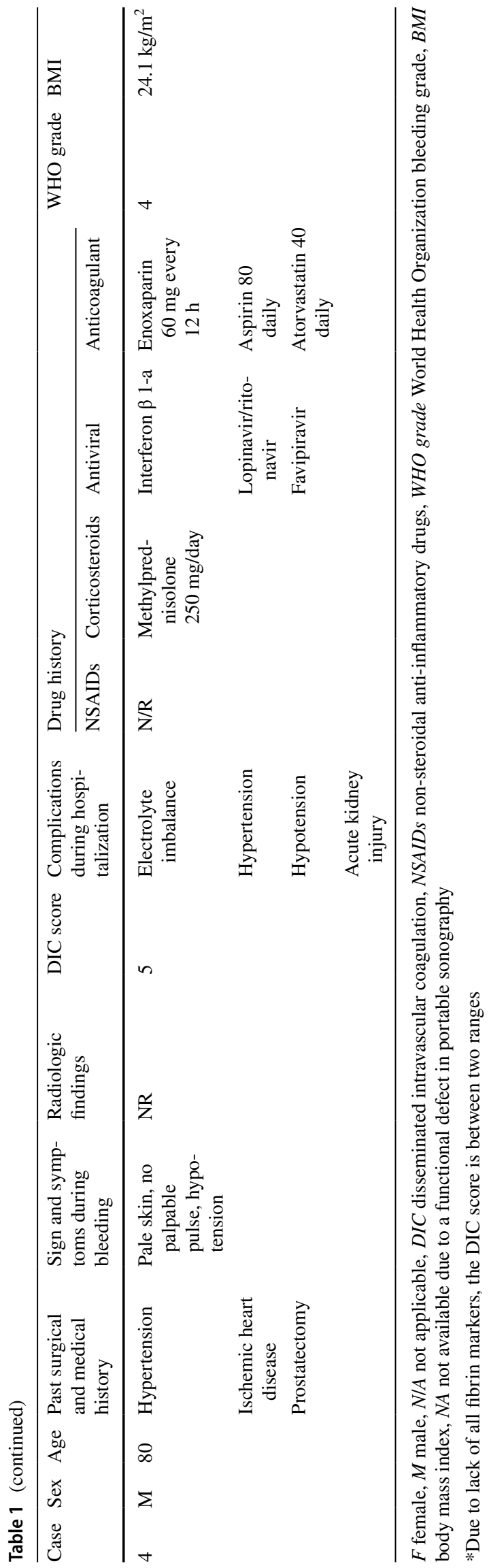

hematoma) (Fig. 1b) and second sonography to observe the changes in the size of hematomas. She gets two more units of pack cell and 4 units of FFP and was transferred to the operation room due to increases in her hematoma detected with sonography.

In the operation room, the patient was packed before finding her all bleeding site due to becoming unstable during the surgery and she did not take her consciousness after that. The next day, although her hemoglobin was corrected and she was stable, she had been become asystole and died.

\section{Case 3}

A 59-year-old man without a history of any specific disease was transferred from another site to our hospital due to the worsening of his condition. His symptoms had been started 2 weeks before his transition and he was hospitalized in the referred site for 4 days. He had experience dyspnea, non-productive coughs, chills, headaches, and pleuritic chest pain. In the emergency department enoxaparin, methylprednisolone, remdesivir, ciprofloxacin, cefepime, famotidine, and other treatments had been administered. In his CTA, the filling defects in 3 lobar arteries in the inferior right lobe and 2-3 segmental arteries in the inferior lobes of both lungs representing PTE were observed. Then he had been transferred to ICU due to his condition and oxygen therapy with NIV and hemoperfusion (3 times) had been started for him. He had good clinical improvements and the dosage of his corticosteroid was reduced to $60 \mathrm{mg} /$ day until the next 7 days. On his 8th day of admission, warfarin was started for him. The next day he had been discharged from ICU and the other day the pains in his right lower limb made us consider DVT for him but there were not any signs of it in color Doppler sonography. The amount of his urination was also reduced while in the physical exam it appears that his bladder was distended. His Foley catheter was changed and the suprapubic catheter was tried which was not successful. $4 \mathrm{~h}$ later Cullen's sign appeared in his suprapubic and periumbilical region and he had a reduction in his blood pressure with a sudden reduction in his hemoglobin from 12.3 to 6.7. In portable echocardiography, there was not any sign of left ventricular systolic. In portable sonography, free fluid in the abdomen and pelvic cavity (Table 1) was seen with an empty bladder. We have administered 4 units of FFP and then $30 \mathrm{mg}$ protamine sulfate but we could not stabilize his vital signs in the next $10 \mathrm{~h}$ even by vasopressors and transfusion and as result, he died while we couldn't take an abdominal CT-Scan and send him to the operation room.

\section{Case 4}

An 80-year-old man with productive coughs, dyspnea, and anorexia that had been started 10 days before was admitted 
Fig. 1 a Case 1 abdominal CTScan with contrast representing hemoperitoneum around the liver, spleen (presented cut). b Case 2, abdominal CT-Scan with contrast representing in this cut large hematoma in the pelvic cavity $(163 * 141 \mathrm{~mm})$ and right rectus muscle sheath hematoma $(41 * 68 \mathrm{~mm})$
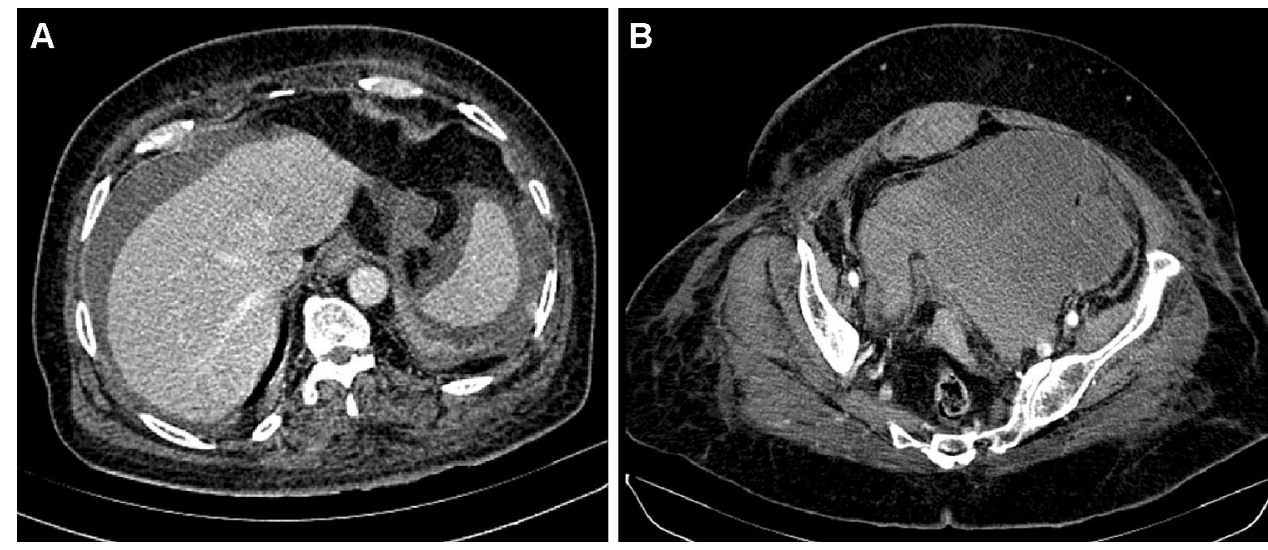

to the emergency department of Shohada hospital. He was an afebrile man with fine crackles in the base of his lungs and oxygen saturation of $70 \%$ in ambient air. His hypoxia caused us to use NIV and a non-rebreather mask in between for him. $\mathrm{He}$ had an incomplete right bundle branch block in ECG and pulmonary hypertension (PAP: $35-40 \mathrm{mmHg}$ ) in echocardiography. Due to his acute renal failure, we performed sonography of the abdomen and kidney to roll out post-renal causes. In his sonography, there were several stones in his non-inflamed gallbladder and mild hydronephrosis that caused us to take an abdominal CT-Scan of him representing mild left lung pleural effusion which was not present before, left kidney hydronephrosis, gallstones, and a suspicious stone in the distal third of left ureter near iliac vessels or vascular calcification there. His high level of D-dimer (1646) caused us to perform CTA of the lungs for him that there were not any signs of massive PTE in it. 15 days after his admission he had a sudden reduction in blood pressure, distended abdomen, pale skin. There were also decreases in the level of hemoglobin and DIC was also suspected (FDP: 4, D-dimer: 942, fibrinogen: 186, Plt: 64,000, DIC score: 5). Although he got 4 units of pack cell and his heart function was normal according to the portable echocardiography, he had died in the morning of the next day.

\section{Discussion and conclusions}

Spontaneous intraperitoneal bleeding is a rare condition with different etiologies; clinical signs include sudden abdominal pain and distention, hypovolemic shock, and changing the color of the skin around the umbilicus and flank reign. The source of the bleeding could be varied from hepatic tumors to splenic, gastrointestinal, visceral artery abnormalities and gynecologic conditions [4]. COVID-19-associated coagulopathies showed unique characteristics; during this infection, patients are at higher risk of developing arterial thrombosis and venous thromboembolism than those with sepsis-induced coagulopathy and DIC. Studies also showed a much lower occurrence of DIC in COVID-19 than sepsis patients, but besides this when the disease progresses from localized lung infection to its systemic phase, the characteristics become more and more similar with DIC [1]. Although we did not check all fibrin markers in cases 2 and 3 , the presented cases' DIC scores were not suggestive for overt DIC (Table 1) [5] which accompanies unique coagulopathy features of COVID-19.

Hemophagocytic lymphohistiocytosis (HLH) could cause coagulation disorders leading to hemorrhage [6] and there is some evidence that hyper-inflammation state could induce HLH in COVID-19 patients [7], but clinical manifestation and laboratory markers (fever, splenomegaly, decreased counts in two cell lines, hypertriglyceridemia and/or hypofibrinogenemia, and hemophagocytosis) of our patients did not make this diagnosis as the $\mathrm{H}$-score for our patient were less than 90. Catastrophic APS is a rare autoimmune disease that could cause coagulation disturbance by increasing aPTT and there is some controversial evidence about its occurrence in critically ill patients with COVID-19 [8, 9]; there are also two cases of COVID-19 bilateral adrenal hemorrhage that one of them had a history of antiphospholipid syndrome and the other one had a suspected history of autoimmune diseases $[10,11]$, however, unfortunately, we did not check markers of this disease but none of our patients developed notable prolonged PTT.

Severe COVID-19 infections could also cause thrombotic microangiopathy (TMA) which is characterized by decreases in hemoglobin, increased lactate dehydrogenase (LDH), increased bilirubin, decreased haptoglobin, and appearance of schistocytes in peripheral blood smear [12]. TMA could also cause major bleeding events [13] and as the presented cases had only decreased in hemoglobin and increased LDH, this would not be the first choice underlying reason for their bleeding and even these changes could be iatrogenic due to several blood samplings.

The occurrence of VTE even in those receiving the full dose of anticoagulants and increased major bleeding events in these patients showed the importance of 
administration of an appropriate dose of anticoagulants, as high doses of them are not suitable for microangiopathy too [2]. A retrospective study on 4389 hospitalized patients showed that AT has reduced mortality and intubation rates in COVID-19 patients. In that study the rate of major bleeding in patients with a prophylactic dose of anticoagulant was approximately the same as those who did not get AT, these numbers were higher in patients who get a therapeutic dose of AT. All these data show a lack of high-quality evidence on the dosage of AT in COVID19 patients and well-conducted randomized clinical trials are needed [14]. High dose of anticoagulant in case 2 and 4 due to high level of D-dimer and their clinical evaluation seems to have harmed their prognosis, but there is also some evidence that reminds us hemorrhagic events in COVID-19 patients are more complicated to blame only AT; a study showed that GI bleedings in COVID-19 patients were not associated with anticoagulant therapies and even prophylaxes with histamine receptor blockers or proton pump inhibitors did not decrease its prevalence which shows a non-acid mediated cause of bleeding in these patients [15]. This evidence shows that there are some other possible mechanisms for bleeding events; nevertheless, a possible solution that is needed to be investigated more is Nafamostat mesylate, an inhibitor of plasmin, thrombin, and trypsin, that could be a choice for AT in high-risk patients as it does not have hemorrhagic side effects even at high doses [3].

New research that showed the potent role of remdesivir to inhibit carboxylesterase-2 showed that iatrogenic interventions could cause some of these serious adverse effects [16]. Co-administration of antiviral drugs with oral anticoagulants in COVID-19 patients increases the plasma concentration of anticoagulants [3]; this could be the reason for some hemorrhagic complications. Another example is corticosteroids, these drugs could slightly increase the incidence of GI bleedings [17] and a study showed that chronic systemic corticosteroids increase the risk of major bleeding events and tamponade in those who underwent aortic valve implantation [18]; besides this, several drug classes could also initiate drug-induced DIC [19] that their list is too long to be mentioned in this article and more studies are needed in this field as we use some newly administered drugs in COVID-19 and this might be the adverse effect of them.

Finally, this case series besides other intraperitoneal bleeding reports in COVID-19 patients [10, 11, 20, 21] demonstrated that COVID-19 coagulopathy and AT should be studied more. To reduce the disease mortality and morbidity investigations on the pathophysiology of COVID-19-associated coagulopathy and finding the best intervention to prevent or treat it is needed.
Author contributions VR: Literature search, data collection, writing the manuscript; ZL: Literature search, review of the manuscript; MD: Literature search, reporting the radiologic findings; MM: Literature search, data collection, review of the manuscript; FR: Literature search, review of the manuscript and final decision to submit for publication.

Funding No funding was received for this study.

\section{Declarations}

Conflict of interest The authors declare no conflicts of interest for this work.

Ethical approval This case series was approved by the Local Ethics Committee of Shahrekord University of Medical Sciences (Registration Number IR.SKUMS.REC.1399.257).

\section{References}

1. Iba T, Levy JH, Connors JM, Warkentin TE, Thachil J, Levi M (2020) The unique characteristics of COVID-19 coagulopathy. Crit Care 24(1): 1-8

2. Chowdhury JF, Moores LK, Connors JM (2020) Anticoagulation in hospitalized patients with COVID-19. N Engl J Med 383(17):1675-1678

3. Dorgalaleh A (2020) Maintaining hemostasis and preventing thrombosis in COVID-19-Part I: bleeding and bleeding risk in COVID-19. In: Seminars in thrombosis and hemostasis, 2020, vol 7. Thieme Medical Publishers, p 815

4. Mortele KJ, Cantisani V, Brown DL, Ros PR (2003) Spontaneous intraperitoneal hemorrhage: imaging features. Radiol Clin 41(6): 1183-1201

5. Levi M, Toh CH, Thachil J, Watson HG (2009) Guidelines for the diagnosis and management of disseminated intravascular coagulation. British Committee for Standards in Haematology. Br J Haematol 145(1):24-33. https://doi.org/10.1111/j.1365-2141.2009. 07600.x

6. Valade S, Mariotte E, Azoulay E (2020) Coagulation disorders in hemophagocytic lymphohistiocytosis/macrophage activation syndrome. Crit Care Clin 36(2):415-426

7. Prilutskiy A, Kritselis M, Shevtsov A, Yambayev I, Vadlamudi C, Zhao Q, Kataria Y, Sarosiek SR, Lerner A, Sloan JM (2020) SARS-CoV-2 infection-associated hemophagocytic lymphohistiocytosis: an autopsy series with clinical and laboratory correlation. Am J Clin Pathol 154(4):466-474

8. Zhang Y, Xiao M, Zhang S, Xia P, Cao W, Jiang W, Chen H, Ding $\mathrm{X}$, Zhao H, Zhang H (2020) Coagulopathy and antiphospholipid antibodies in patients with COVID-19. N Engl J Med 382(17):e38

9. Borghi MO, Beltagy A, Garrafa E, Curreli D, Cecchini G, Bodio C, Grossi C, Blengino S, Tincani A, Franceschini F (2020) Antiphospholipid antibodies in COVID-19 are different from those detectable in the anti-phospholipid syndrome. Front Immunol 11:2692

10. Frankel M, Feldman I, Levine M, Frank Y, Bogot NR, Benjaminov O, Kurd R, Breuer GS, Munter G (2020) Bilateral adrenal hemorrhage in coronavirus disease 2019 patient: a case report. J Clin Endocrinol Metab 105(12):3745-3749

11. Álvarez-Troncoso J, Larrauri MZ, Vega MDM, Vallano RG, Peláez EP, Rojas-Marcos PM, Martín-Luengo F, Del Campo PL, Gil CRH, Esteban ET (2020) Case report: COVID-19 with bilateral adrenal hemorrhage. Am J Trop Med Hyg 103(3):1156-1157 
12. Gavriilaki E, Brodsky RA (2020) Severe COVID-19 infection and thrombotic microangiopathy: success does not come easily. $\mathrm{Br} \mathrm{J}$ Haematol 189(6):e227-e230

13. Wang X, Liu C-Y, Yang Y, Zou G-M, Zhuo L, Han S-H, Li W-G (2020) Acute kidney injuries induced by thrombotic microangiopathy following severe hemorrhage in puerperants: a case series and literature review

14. Nadkarni GN, Lala A, Bagiella E, Chang HL, Moreno PR, Pujadas E, Arvind V, Bose S, Charney AW, Chen MD (2020) Anticoagulation, bleeding, mortality, and pathology in hospitalized patients with COVID-19. J Am Coll Cardiol 76(16):1815-1826

15. Trindade AJ, Izard S, Coppa K, Hirsch JS, Lee C, Satapathy SK, Consortium NCR, Barish MA, Molmenti EP, Bernstein DE (2020) Gastrointestinal bleeding in hospitalized COVID-19 patients: a propensity score matched cohort study. J Intern Med. https://doi. org/10.1111/joim. 13232

16. Shen Y, Eades W, Yan B (2020) Remdesivir potently inhibits carboxylesterase-2 through covalent modifications: signifying strong drug-drug interactions. Fundam Clin Pharmacol. https://doi.org/ 10.1111/fcp. 12643

17. Butler E, Møller MH, Cook O, Granholm A, Penketh J, Rygård SL, Aneman A, Perner A (2019) The effect of systemic corticosteroids on the incidence of gastrointestinal bleeding in critically ill adults: a systematic review with meta-analysis. Intensive Care Med 45(11):1-10
18. Gautier A, Urena M, Chong-Nguyen C, Fischer Q, Abtan J, Carrasco JL, Brochet E, Iung B, Himbert D (2020) Outcomes of transcatheter aortic valve implantation in patients receiving chronic systemic corticosteroid treatment. Am J Cardiol 130:108-114

19. Bonaldo G, Vaccheri A, Melis M, Motola D (2020) Drug-induced disseminated intravascular coagulation: a pharmacovigilance study on World Health Organization's database. J Thromb Thrombolysis 50(4):763-771

20. Karki S, Rawal SB, Malla S, Rayamajhi J, Thapa BB (2020) A case report on spontaneous hemoperitoneum in COVID-19 patient. Int J Surg Case Rep 75:211-213

21. Al-Samkari H, Karp Leaf RS, Dzik WH, Carlson JC, Fogerty AE, Waheed A, Goodarzi K, Bendapudi PK, Bornikova L, Gupta S (2020) COVID-19 and coagulation: bleeding and thrombotic manifestations of SARS-CoV-2 infection. Blood J Am Soc Hematol 136(4):489-500

Publisher's Note Springer Nature remains neutral with regard to jurisdictional claims in published maps and institutional affiliations. 